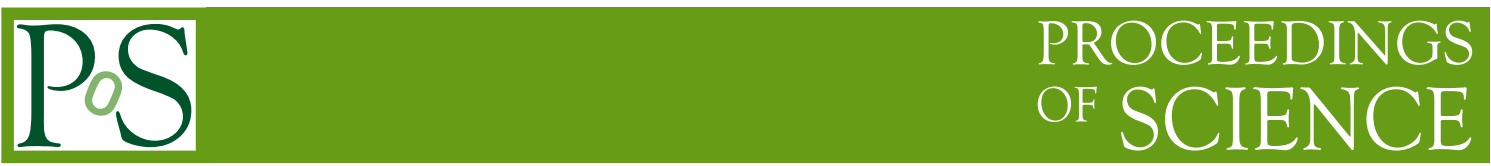

Sixth Annual Conference on Large Hadron Collider Physics (LHCP2018)

4-9 June 2018

Bologna, Italy

\title{
New results on collectivity in small systems with CMS
}

\author{
Jovan Milosevic ${ }^{*}$ on behalf of the CMS Collaboration \\ Vinca Institute of Nuclear Sciences, University of Belgrade, Belgrade, 11001, Serbia \\ E-mail: Jovan.Milosevic@cern.ch
}

The elliptic $\left(v_{2}\right)$ flow of prompt $\mathrm{D}^{0}$ mesons, together with $\mathrm{K}_{S}^{0}, \Lambda, \Xi$, and $\Omega$ in high-multiplicity $\mathrm{pPb}$ collisions at $8.16 \mathrm{TeV}$, is presented. The data are collected with the CMS detector at the LHC from the rapidity interval $-1.46<y_{c m}<0.54$ and transverse momentum range $1.5<\mathrm{p}_{\mathrm{T}}<8 \mathrm{GeV}$. The results are compared to those from the $30-50 \%$ centrality $\mathrm{PbPb}$ data at $5.02 \mathrm{TeV}$. A massordering behavior with a stronger $v_{2}$ of lighter species for $\mathrm{p}_{\mathrm{T}}<2 \mathrm{GeV}$ is observed. The $v_{2}$ scaled to the number of constituent quarks vs the transverse kinetic energy per constituent quark shows that the $\mathrm{D}^{0}$ scaled $v_{2}$ values in $\mathrm{pPb}$ collisions are smaller than those of light-flavor particles. Such an effect is not observed in $\mathrm{PbPb}$ collisions indicating a weaker collectivity of charm quarks than the one of light-flavor quarks, and a weaker coupling between heavy quarks and a possibly formed quark-gluon plasma droplet. The $v_{2}$ of prompt $\mathrm{J} / \psi$ mesons in high-multiplicity $\mathrm{pPb}$ collisions at $8.16 \mathrm{TeV}$ is also measured. The $\mathrm{J} / \psi$ mesons are reconstructed via the dimuon channel with 1.4 $<|y|<2.4$ and $2<\mathrm{p}_{\mathrm{T}}<8 \mathrm{GeV}$. This observation provides the most direct evidence for the presence of charm quark collectivity in $\mathrm{pPb}$ collisions. Comparison with $v_{2}$ of $\mathrm{D}^{0}$ and strange hadrons allows a strong constraints on the dynamics of charm quarks produced in small hadronic collision systems with high multiplicities. Additionally, the standard and the subevent cumulant method is used to measure correlations between the $v_{2}$ and triangular $\left(v_{3}\right)$ flow, and between the $v_{2}$ and quadrupole $\left(v_{4}\right)$ flow in $\mathrm{pPb}$ collisions up to very low multiplicities. At high multiplicities, all methods give similar results showing that non-flow is not the dominant in this region, while at low multiplicities, the subevent method allows to strongly suppress the non-flow contribution and reveals the pure collectivity.

\footnotetext{
*Speaker.

$\dagger$ Thanking to financial support from MESTD, Republic of Serbia.
} 


\section{Introduction}

Particles emitted in relativistic nucleus-nucleus collisions exhibit collective azimuthal $(\phi)$ correlations which are long-range in pseudorapidity $(\eta)$. These correlations suggest the formation of a strongly interacting quark-gluon plasma (QGP) that exhibits nearly ideal hydrodynamic behavior [1,2]. Similar long-range collective azimuthal correlations are also observed in highmultiplicity proton-lead ( $\mathrm{pPb}$ ) events [3]. A question appears of whether a fluid-like QGP is created also in such a small system. Experimental measurements in these small systems are consistent with the hydrodynamic expansion of a tiny QGP droplet, but an alternative scenario based on gluon saturation in the initial state also claims ability to describe the main features of these correlations [4].

In the early stages of the hadronic collision, charm $(c)$ and bottom $(b)$ quarks are produced via hard scatterings. Thus, they could be used to study the properties and dynamics of the QGP through its entire evolution [5]. The observation of the elliptic flow $\left(v_{2}\right)$ of prompt $\mathrm{D}^{0}$ mesons in $\mathrm{pPb}$ collisions provided an evidence for $c$ quark collectivity in a small system [6]. The $v_{2}$ of prompt $\mathrm{D}^{0}$ mesons has somewhat smaller magnitude with respect to the $v_{2}$ produced by particles formed from light $u$ and $d$ quarks suggesting a weaker collective motion of $c$ quarks relative to that of the bulk medium. As the $\mathrm{D}^{0}$ meson is formed from both a light and a $c$ quark, the relative contribution of different flavor quarks to the prompt $\mathrm{D}^{0} v_{2}$ signal is not fully constrained. Thus, the observation of the $v_{2}$ signal for $\mathrm{J} / \psi$ mesons in $\mathrm{pPb}$ collisions can provide the most direct evidence of $c$ quark collectivity and impose new constraints on the collective dynamics of heavy quarks and their interaction with possibly formed QGP in small systems.

Additionally, in order to understand the onset of collective behavior from high- to low-multiplicity events in small systems, a subevent symmetric four-particle cumulant technique [7] has been performed using $8.16 \mathrm{TeV} \mathrm{pPb}$ data. For low-multiplicity events with $N_{t r k}^{o f f l i n e} \leq 80$, this method has been used to suppress non-collective contribution coming from few-particle correlations which arise from jets and thus to reveals a pure long-range azimuthal correlations up to events with $N_{t r k}^{o f f l i n e} \geq 50$. The method also allows to study event-by-event correlations among the $v_{2}$, and $v_{3}$ and $v_{4}$ Fourier harmonics, and to highlights its collective nature $[3,8,9]$. Namely, the correlation data are similar to those observed in $\mathrm{PbPb}$ collisions, where a negative correlation is found between $v_{2}$ and $v_{3}$ harmonics, while the correlation is positive between $v_{2}$ and $v_{4}$. This observation further supports the hydrodynamic origin of collective correlations in small systems.

\section{Experiment and data used}

The central feature of the CMS apparatus is a superconducting solenoid of $6 \mathrm{~m}$ internal diameter, providing a magnetic field of 3.8T. Within the solenoid volume are a silicon pixel and strip tracker, a lead tungstate crystal electromagnetic calorimeter (ECAL), and a brass and scintillator hadron calorimeter (HCAL), each composed of a barrel and two endcaps. Forward calorimeters extend the pseudorapidity coverage provided by the barrel and endcap detectors. Muons are detected in gas-ionization chambers embedded in the steel flux-return yoke outside the solenoid. The silicon tracker measures charged particles within the range $|\eta|<2.5$. For charged particles with transverse momentum $1<\mathrm{p}_{\mathrm{T}}<10 \mathrm{GeV}$ and $|\eta|<1.4$, typical track resolution is $1.5 \%$ in $\mathrm{p}_{\mathrm{T}}$ and 25-90 (45-150) $\mu \mathrm{m}$ in the transverse (longitudinal) impact parameter [10]. Muons are measured 
in the $|\eta|<2.4$ range. A more detailed description of the CMS detector, together with a definition of the coordinate system used and the relevant kinematic variables, can be found in Ref. [11].

The $\mathrm{pPb}$ data at $\sqrt{s_{N N}}=8.16 \mathrm{TeV}$ used in these analyses were collected by the CMS detector, and correspond to an integrated luminosity of $186 \mathrm{nb}^{-1}$. The quantity $N_{t r k}^{\text {of }}$ fline is the number of primary charged-particle tracks [10] with $|\eta|<2.4$ and $\mathrm{p}_{\mathrm{T}}>0.4 \mathrm{GeV}$. Events with $N_{t r k}^{\text {offline }}<35$ are used to estimate the contribution of residual non-flow back-to-back jet-like correlations. It is required that the events contain a primary vertex close to the nominal interaction point of the beams, within $15 \mathrm{~cm}$ along the beam direction, and $0.2 \mathrm{~cm}$ in the plane transverse to the beam direction.

Muon candidates are reconstructed either by finding tracks in the muon detectors, and then fitting them together with tracks reconstructed in the silicon tracker, or by extrapolating tracks from the silicon tracker to match a hit on at least one segment of the muon detectors. The muon candidates are also required to pass the identification criteria of the particle-flow algorithm [12].

The $\mathrm{J} / \psi$ meson candidates are formed from $\mu^{+} \mu^{-}$pairs, originating from a common vertex. Due to the long lifetime of $\mathrm{b}$ hadrons compared to that of $\mathrm{J} / \psi$ mesons, nonprompt $\mathrm{J} / \psi$ components can be discriminated by measuring the displacement between the secondary $\mu^{+} \mu^{-}$vertex and the primary collision vertex.

\section{Methods used}

The azimuthal anisotropy of $\mathrm{J} / \psi$ mesons is extracted from the long-range $(|\Delta \eta|>1)$ twoparticle azimuthal correlations, following an identical procedure to that described in Refs. [3, 6, 8].

The symmetric cumulant (SC) is defined as

$$
S C(n, m)=\langle\langle 4\rangle\rangle_{n, m, n, m}-\langle\langle 2\rangle\rangle_{n, n}\langle\langle 2\rangle\rangle_{m, m}
$$

where 2- and 4-particle correlators are defined as

$$
\langle\langle 2\rangle\rangle_{n, n}=\left\langle\left\langle e^{i\left(n \phi_{1}-n \phi_{2}\right)}\right\rangle\right\rangle,\langle\langle 4\rangle\rangle_{n, m, n, m}=\left\langle\left\langle e^{i\left(n \phi_{1}+m \phi_{2}-n \phi_{3}-m \phi_{4}\right)}\right\rangle\right\rangle
$$

where $n$ and $m$ correspond to different harmonic order.

The $\langle\langle\ldots\rangle\rangle$ denotes the averaging over all events and over all particles of interest. The two-, three- and four-subevent cumulant $\left(S C_{s u b}\right)$ are then defined as

$$
\begin{gathered}
S C_{2 s u b}(n, m)=\left\langle\langle 4\rangle_{n, m \mid n, m}^{a, a \mid b^{*} b^{*}}\right\rangle-\left\langle\langle 2\rangle_{n \mid n}^{a \mid b^{*}}\right\rangle\left\langle\langle 2\rangle_{m \mid m}^{a \mid b^{*}}\right\rangle, \\
S C_{3 s u b}(n, m)=\left\langle\langle 4\rangle_{n|m, n| m}^{a^{*}|b b| c^{*}}\right\rangle-\left\langle\langle 2\rangle_{n \mid n}^{b \mid a^{*}}\right\rangle\left\langle\langle 2\rangle_{m \mid m}^{b \mid c^{*}}\right\rangle, \\
S C_{4 s u b}(n, m)=\left\langle\langle 4\rangle_{n|m| n \mid m}^{a^{*}|b| c^{*} \mid d^{*}}\right\rangle-\left\langle\langle 2\rangle_{n \mid n}^{a \mid c^{*}}\right\rangle\left\langle\langle 2\rangle_{m \mid m}^{b \mid d^{*}}\right\rangle .
\end{gathered}
$$

where $a, b, c$ and $d$ denote the particles chosen in each subevent spanned over certain $\eta$ window (for more details see [13]).

\section{Results}

The elliptic flow results corrected for residual jet correlations $\left(v_{2}^{\text {sub }}\right)$ are shown in Fig. 1 (top left) for prompt $\mathrm{D}^{0}$ mesons and for strange hadrons as functions of $\mathrm{p}_{\mathrm{T}}$ for high-multiplicity $\mathrm{pPb}$ 
collisions. The prompt $\mathrm{D}^{0} v_{2}^{\text {sub }}$ shows a $\mathrm{p}_{\mathrm{T}}$-dependence similar to those seen for particles made from light flavor quarks. In order to compare qualitatively the obtained results, in the top right panel of Fig. 1 are shown corresponding results extracted from $\mathrm{PbPb}$ collisions within centrality range of 30-50\%. Collectivity at the partonic level is investigated by studying the scaling properties of $v_{2}^{s u b}$ divided by the number of constituent quarks $\left(n_{q}\right)$ as a function of transverse kinetic energy per constituent quark, $K E_{T} / n_{q}$, for all measured particles. The corresponding plots for $\mathrm{pPb}$ (bottom left) and $\mathrm{PbPb}$ (bottom right panel) collisions are shown in Fig. 1 . In $\mathrm{pPb}$ collisions, the $v_{2}^{\text {sub }} / n_{q}$ for strange hadrons tend to follow a common trend for $0.5<K E_{T} / n_{q}<1.5 \mathrm{GeV}$, while the $\mathrm{D}^{0} v_{2}^{\text {sub }} / n_{q}$ has smaller value. This could suggest that the collective behavior of $c$ quarks is weaker than that of the light-flavor $u, d$ and $s$ quarks. For $K E_{T} / n_{q}$ above $1.5 \mathrm{GeV}$, no clear scaling of $v_{2}^{\text {sub }} / n_{q}$ between mesons and baryons is observed. This is qualitatively different in the larger $\mathrm{PbPb}$ collision system with centrality between 30 and 50\% where all particles show tendency to follow a universal trend.
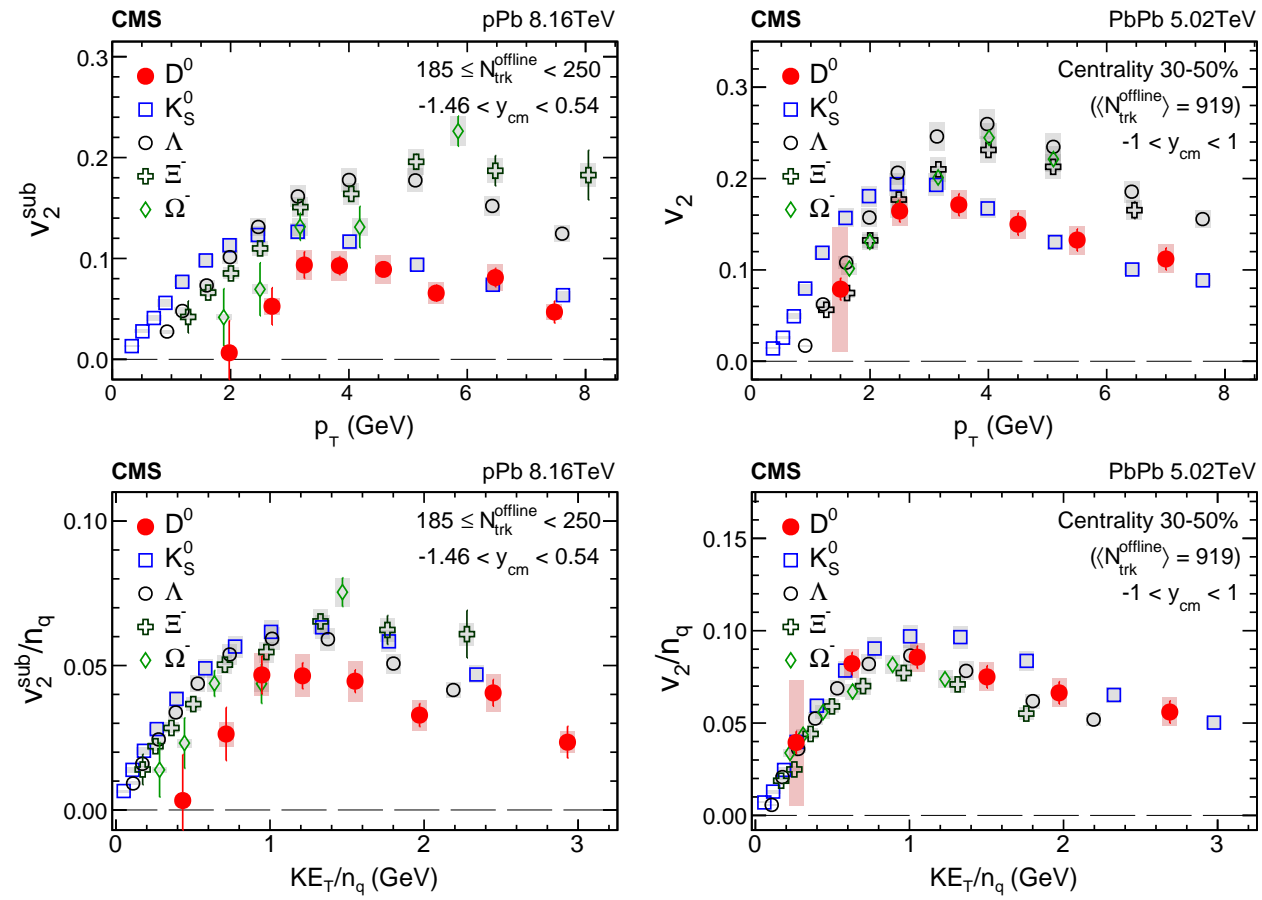

Figure 1: Top: prompt $\mathrm{D}^{0}$ meson and strange hadrons $v_{2}^{\text {sub }} v s \mathrm{p}_{\mathrm{T}}$ in $\mathrm{pPb}$ collisions at $\sqrt{s_{N N}}=8.16 \mathrm{TeV}$ with $185 \leq N_{t r k}<250$ (left) and $\mathrm{PbPb}$ collisions at $\sqrt{s_{N N}}=5.02 \mathrm{TeV}$ with centrality of 30-50\% (right) [6]. Bottom: the $v_{2}^{\text {sub }}$ scaled to the number of constituent quarks [6]. The $\mathrm{D}^{0} v_{2}^{\text {sub }}$ data in $\mathrm{PbPb}$ collisions are taken from Ref. [14]. The error bars (shaded areas) correspond to the statistical (systematic) uncertainties.

The $v_{2}^{s u b}$ values for prompt $\mathrm{J} / \psi$ mesons as a function of $\mathrm{p}_{\mathrm{T}}$ in high-multiplicity $\mathrm{pPb}$ collisions are shown in Fig. 2 (top) and compared to $\mathrm{K}_{S}^{0}$ and $\mathrm{D}^{0}$ meson results. As in the case of $\mathrm{D}^{0}$ mesons, the $\mathrm{J} / \psi v_{2}^{s u b}$ exhibits a characteristic $\mathrm{p}_{\mathrm{T}}$ dependence: first it rises, reaches a maximum and then decreases going to higher $\mathrm{p}_{\mathrm{T}}$ values. In the context of the quark coalescence model, in Fig. 2 (bottom) are shown corresponding $v_{2}^{\text {sub }}$ values scaled to the $n_{q}$ and plotted $v s K E_{T} / n_{q}$ to represent the collectivity at the partonic level. Over the full $K E_{T} / n_{q}$ range, the scaled $v_{2}^{\text {sub }}$ for prompt $\mathrm{D}^{0}$ and $\mathrm{J} / \psi$ mesons is consistently below that of $\mathrm{K}_{S}^{0}$ meson. However, no definitive conclusion can yet be claimed based on current experimental uncertainties. 

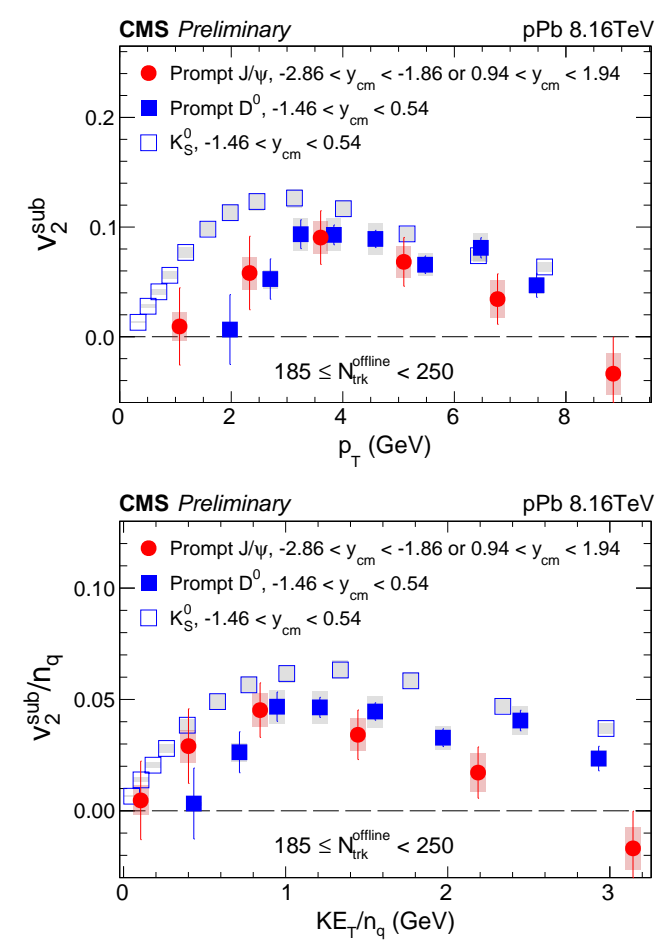

Figure 2: Top: Prompt $\mathrm{J} / \psi$ and $\mathrm{D}^{0}$, as well as $\mathrm{K}_{S}^{0} v_{2}^{s u b} v s \mathrm{p}_{\mathrm{T}}$ for $\mathrm{pPb}$ collisions at $\sqrt{s_{N N}}=8.16 \mathrm{TeV}$ with $185 \leq N_{t r k}<250$ [15]. Bottom: the $v_{2}^{s u b}$ scaled to the number of constituent quarks [15]. The $\mathrm{K}_{S}^{0}$ and $\mathrm{D}^{0} v_{2}^{\text {sub }}$ data are taken from Ref. [6]. The error bars (shaded areas) correspond to statistical (systematic) uncertainties.

Symmetric cumulants $S C(2,3)$ and $S C(2,4)$ for 2-, 3- and 4-subevents as a function of multiplicity in $\mathrm{pPb}$ collisions are shown in Fig. 3. For a comparison, in the same figure are also shown corresponding results with no subevents. For $N_{t r k}^{o f f l i n e}<80$, both $S C(2,3)$ and $S C(2,4)$ diverge toward positive values in the no subevent case, likely because of a dominant contribution of few-particle correlations. But, in the case of using the subevent method, this trend is strongly suppressed. For $N_{t r k}^{o f f l i n e}>50$ a clear negative values of $\operatorname{SC}(2,3)$ are observed, while $S C(2,4)$ values are positive. These observations are similar to what was seen in $\mathrm{PbPb}$ collisions [16], and provide further evidence of collectivity observed in small systems down to events with $N_{t r k}^{o f f l i n e}$ of $\approx 50$.

\section{Summary}

Significant $v_{2}$ values of prompt $\mathrm{D}^{0}$ and $\mathrm{J} / \psi$ mesons in high-multiplicity pPb collisions at $8.16 \mathrm{TeV}$ are measured. This observation provides an evidence for $c$ quark collectivity in small collision system. The collectivity is weaker with respect to the one observed for light flavor quarks, unlike what is found in $\mathrm{PbPb}$ collisions. That provides strong constraints on the heavy quarks dynamics in small systems. The $S C_{\text {sub }}(2,3)$ and $S C_{\text {sub }}(2,4)$ are measured in $\mathrm{pPb}$ collisions at 8.16 TeV. An anti-correlation is observed between the elliptic flow $v_{2}$ and Fourier harmonic $v_{3}$, while $v_{2}$ and Fourier harmonic $v_{4}$ are positively correlated. At high multiplicities, standard and $S C_{\text {sub }}$ give similar results for $S C(2,3)$ suggesting that non-flow is not dominant in this region, 

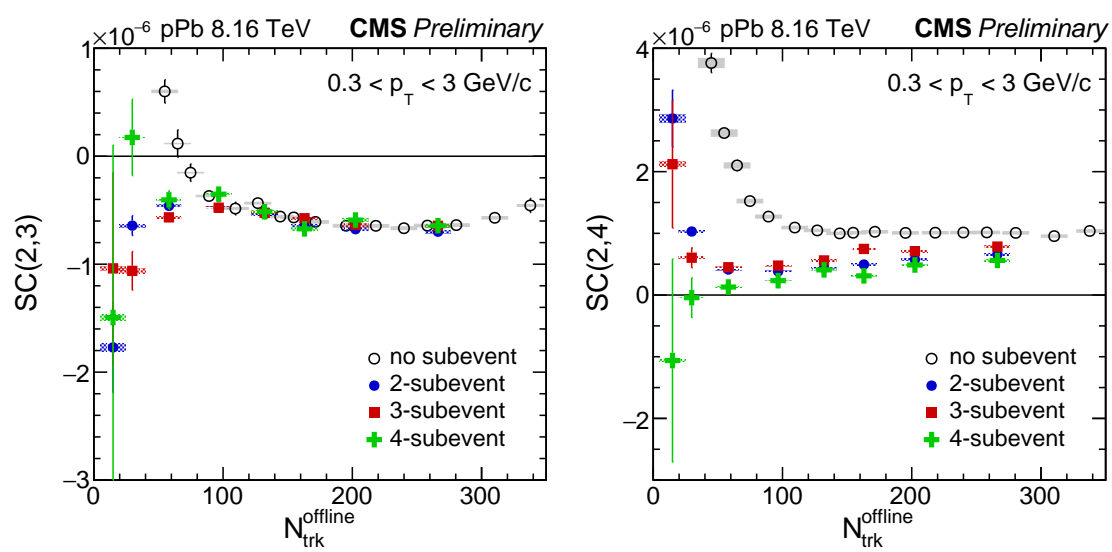

Figure 3: $S C(2,3)$ and $S C(2,4)$ v $N_{t r k}^{\text {offline }}$ from 2-, 3- and 4-subevents method [13]. Results taken from the Ref. [16] with no subevents are also shown. Shaded areas denote the systematic uncertainties.

while $S C(2,4)$ show a different behavior for different numbers of subevents, which emphasizes the sensitivity of $S C(2,4)$. For $50<N_{t r k}^{\text {offline }}<80$, the subevent method suppresses the non-flow contribution and reveals the harmonic correlation from collectivity.

\section{References}

[1] J.-Y. Ollitrault, Phys. Rev. D 46 (1992) 229

[2] U. Heinz and R. Snellings, Ann. Rev. Nucl. Part. Sci. 63 (2013) 123

[3] V. Khachatryan et al., CMS Collaboration, Phys. Rev. Lett. 115 (2015) 012301

[4] K. Dusling, W. Li, and B. Schenke, Int. J. Mod. Phys. E25 (2016) 1630002

[5] P. Braun-Munzinger, J. Phys. G34 (2007) S471

[6] A. M. Sirunyan et al., CMS Collaboration, Phys. Rev. Lett. 121 (2018) 082301

[7] J. Adam et al., ALICE Collaboration, Phys. Rev. Lett. 117 (2016) 182301

[8] V. Khachatryan et al., CMS Collaboration, Phys. Lett. B765 (2017) 193

[9] M. Aaboud et al., ATLAS Collaboration, Eur. Phys. J. C77 (2017) 428

[10] S. Chatrchyan et al., CMS Collaboration, JINST 9 (2014) P10009

[11] S. Chatrchyan et al., CMS Collaboration, JINST 3 (2008) S08004

[12] A. M. Sirunyan et al., CMS Collaboration, JINST 12 (2017) P10003

[13] CMS Collaboration, CMS-PAS-HIN-18-015, https://cds.cern.ch/record/2318291?ln=en, (2018)

[14] A. M. Sirunyan et al., CMS Collaboration, Phys. Rev. Lett. 120 (2018) 202301

[15] CMS Collaboration, CMS-PAS-HIN-18-010, https://cds.cern.ch/record/2318326?ln=en, (2018)

[16] A. M. Sirunyan et al., CMS Collaboration, Phys. Rev. Lett. 120 (2018) 092301 